\title{
Self-generated neural activity: models and perspective Claudius Gros
}

\author{
Address: Department of Physics, Frankfurt University, Frankfurt a.M., 60054, Germany \\ Email: Claudius Gros - gros07@itp.uni-frankfurt.de
}

from Eighteenth Annual Computational Neuroscience Meeting: CNS*2009

Berlin, Germany. 18-23 July 2009

Published: 13 July 2009

BMC Neuroscience 2009, I0(Suppl I):P272 doi:10.| |86/|47|-2202-I0-SI-P272

This abstract is available from: http://www.biomedcentral.com/I47I-2202/I0/SI/P272

(c) 2009 Gros; licensee BioMed Central Ltd.

The brain is autonomously active and this self-sustained neural activity is in general modulated, but not driven, by the sensory input data stream $[1,2]$. Traditionally one has regarded this eigendynamics as resulting from inter-modular recurrent neural activity [3]. Understanding the basic modules for cognitive computation is, in this view, the primary focus of research and the overall neural dynamics would be determined by the the topology of the intermodular pathways. Here we examine an alternative point of view, asking whether certain aspects of the neural eigendynamics have a central functional role for overall cognitive computation $[4,5]$.

Transiently stable neural activity is regularly observed on the cognitive time-scale of $80-100 \mathrm{~ms}$, with indications that neural competition [6] plays an important role in the selection of the transiently stable neural ensembles [7], also denoted winning coalitions [8]. We report on a theory approach which implements these two principles, transient-state dynamics and neural competition, in terms of an associative neural network with clique encoding [9].

A cognitive system [10] with a non-trivial internal eigendynamics has two seemingly contrasting tasks to fulfill. The internal processes need to be regular and not chaotic on one side, but sensitive to the afferent sensory stimuli on the other side. We show, that these two contrasting demands can be reconciled within our approach based on competitive transient-state dynamics, when allowing the sensory stimuli to modulate the competition for the next winning coalition. By testing the system with the bars problem, we find an emerging cognitive capability. Only based on the two basic architectural principles, neural competition and transient-state dynamics, with no explicit algorithmic encoding, the system performs on its own a non-linear independent component analysis of input data stream. The system has rudimentary biological features. All learning is local Hebbian-style, unsupervised and online. It exhibits an ever-ongoing eigendynamics and at no time is the state or the value of synaptic strengths reset or the system restarted; there is no separation between training and performance. We believe that this kind of approach - cognitive computation with autonomously active neural networks - to be an emerging field, relevant both for system neuroscience and synthetic cognitive systems.

\section{References}

I. Ringach DL: States of mind. Nature 2003, 425:9/2-9/3.

2. Fiser J, Chiu C, Weliky M: Small modulation of ongoing cortical dynamics by sensory input during natural vision. Nature 2004, 431:573-578.

3. Arieli A, Sterkin A, Grinvald A, Aertsen A: Dynamics of Ongoing Activity: Explanation of the Large Variability in Evoked Cortical Responses. Science 1996, 273: I868-I87|.

4. Gros C: Cognitive computation with autonomously active neural networks: an emerging field. Cognitive Computation 2009 in press. (a review)

5. Gros C: Emotions, diffusive emotional control and the motivational problem for autonomous cognitive systems. Handbook of Research on Synthetic Emotions and Sociable Robotics: New Applications in Affective Computing and Artificial Intelligence 2009 in press. (a chapter contribution).

6. Fox MD, Snyder AZ, Vincent JL, Corbetta M, Van Essen DC, Raichle ME: The human brain is intrinsically organized into dynamic, anticorrelated functional networks. Proceedings of the National Academy of Sciences 2005, 102:9673-9678.

7. Abeles M, Bergman H, Gat I, Meilijson I, Seidemann E, Tishby N, Vaadia $\mathrm{E}$ : Cortical activity flips among quasi-stationary states. Proceedings of the National Academy of Sciences 1995, 92:86 16-8620.

8. Crick FC, Koch C: A framework for consciousness. Nature Neuroscience 2003, 6:1 19-126. 
9. Gros C: Neural networks with transient state dynamics. New Journal of Physics 2007, 9:109.

10. Gros C: Complex and Adaptive Dynamical Systems, A Primer New York: Springer-Verlag; 2008.

Publish with Bio Med Central and every scientist can read your work free of charge

"BioMed Central will be the most significant development for disseminating the results of biomedical research in our lifetime. " Sir Paul Nurse, Cancer Research UK

Your research papers will be:

- available free of charge to the entire biomedical community

- peer reviewed and published immediately upon acceptance

- cited in PubMed and archived on PubMed Central

- yours - you keep the copyright 